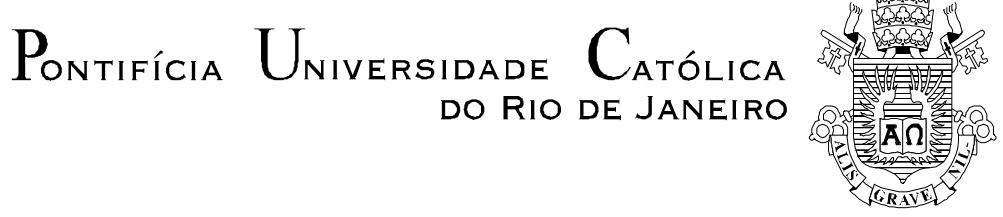

Wagner Saboia de Abreu

\begin{abstract}
Modelagem e Previsão de Preços à Vista de Energia Elétrica e Aplicações no Contexto de Investimentos sob Incerteza
\end{abstract}

\begin{abstract}
Dissertação de Mestrado
Dissertação apresentada como requisito parcial para obtenção de grau de Mestre pelo Programa de Pósgraduação em Engenharia de Produção do Departamento de Engenharia Industrial da PUC-Rio.
\end{abstract}

Orientador: Prof. Carlos Patricio Samanez Co-orientador: Prof. Tara Keshar Nanda Baidya

Rio de Janeiro

Abril de 2012 
Pontifícia Universidade Católica $_{\text {a }}$

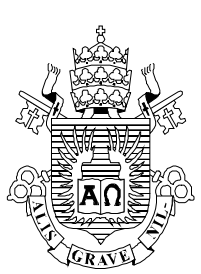

\section{Wagner Saboia de Abreu}

\section{Modelagem e Previsão de Preços à Vista de Energia Elétrica e Aplicações no Contexto de Investimentos sob Incerteza.}

Dissertação apresentada como requisito parcial para obtenção do grau de Mestre pelo Programa de Pósgraduação em Engenharia de Produção do Departamento de Engenharia Industrial do Centro Técnico Científico da PUC-Rio. Aprovada pela Comissão Examinadora abaixo assinada.

Prof. Carlos Patricio Samanez Orientador

Departamento de Engenharia Industrial - PUC-Rio

Prof. Tara Keshar Nanda Baidya

Co-orientador

Departamento de Engenharia Industrial - PUC-Rio

Prof. Marco Antonio Guimarães Dias Departamento de Engenharia Industrial - PUC-Rio

Prof. Juan Guillermo Lazo Lazo Departamento de Engenharia Elétrica - PUC-Rio

Prof. Fernando Antonio Lucena Aiube Departamento de Engenharia Industrial - PUC-Rio

Prof. Jose Eugenio Leal Coordenador Setorial do Centro Técnico Científico - PUC-Rio

Rio de Janeiro, 9 de Abril de 2012 
Todos os direitos reservados. É proibida a reprodução total ou parcial do trabalho sem autorização da universidade, do autor e do orientador.

\section{Wagner Saboia de Abreu}

Graduou-se em Engenharia de Produção (2009) pela Pontifícia Universidade do Rio de Janeiro (PUC-Rio) e em Engenharia Generalista (2008) pela École Centrale Marseille. Atuando principalmente nos seguintes temas: eficiência energética, opções reais, sustentabilidade.

Ficha Catalográfica

Abreu, Wagner Saboia de

Modelagem e previsão de preços à vista de energia elétrica e aplicações no contexto de investimentos sob incerteza / Wagner Saboia de Abreu ; orientador: Carlos Patricio Samanez ; co-orientador: Tara Keshar Nanda Baidya. - 2012.

$111 \mathrm{f}$; $30 \mathrm{~cm}$

Dissertação (mestrado)-Pontifícia Universidade Católica do Rio de Janeiro, Departamento de Engenharia Industrial, 2012.

Inclui bibliografia

1. Engenharia Industrial - Teses. 2. Setor elétrico brasileiro. 3. Comercialização de energia elétrica. 4. Apreçamento de contratos de energia. 5. Método de Monte Carlo. I. Samanez, Carlos Patrício II. Baidya, Tara Keshar Nanda. III. Pontifícia Universidade Católica do Rio de Janeiro. Departamento de Engenharia Industrial. IV. Título.

CDD: 658.5 


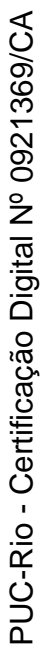

A Deus, sem o qual nada seria possível. 


\section{Agradecimentos}

Aos meus pais, Verônica e Valcinei, pela vida e pela educação;

À Renata, pelo amor, carinho e compreensão, além da revisão do trabalho;

À Camila, minha irmã, e a Diego, seu marido, por todas as conversas de apoio.

Ao Professor Tara Keshar Nanda Baidya, pela orientação, compreensão, paciência e amizade.

Ao Professor Leonardo Lima Gomes, pela ideia que levou a esta dissertação.

Aos Professores Juan Lazo e Fábio Batista, pela revisão e concelhos;

Aos Professores Carlos Patricio Samanez, José Paulo Teixeira, Marco Antonio Guimarães Dias, Fernando Aiube e Kátia Rocha, pelos ensinamentos sem os quais este trabalho não seria possível;

Aos funcionários do DEI, Ana Carolina, Ana Zélia, Celi, Cláudia, Dilza, Eduardo, Fernanda, Gilvan e Isabel, por todo apoio, paciência e ajuda;

Aos colegas, Eduardo, Frances, Priscila, Rafael, Thaís, entre outros, pelo companheirismo e apoio;

À PUC-Rio, por todo apoio durante a graduação e durante mestrado; e Ao CNPq, pelo apoio financeiro. 


\section{Resumo}

Abreu, Wagner Saboia de; Samanez, Carlos Patricio (Orientador); Baidya, Tara Keshar Nanda (Coorientador). Modelagem e Previsão de Preços à Vista de Energia Elétrica e Aplicações no Contexto de Investimentos sob Incerteza. Rio de Janeiro, 2012. 111p. Dissertação de Mestrado Departamento de Engenharia Industrial, Pontifícia Universidade Católica do Rio de Janeiro.

O Setor Elétrico Brasileiro (SEB) passou por uma grande reestruturação, saindo de uma situação de monopólio estatal para uma de desestatização regulamentada. Neste processo, a interação entre os agentes, causada pelas privatizações ocorridas no setor, passou a condicionar a formação dos preços do mercado de energia elétrica e, consequentemente, dos contratos dela derivados. $\mathrm{O}$ presente trabalho coloca a eletricidade no contexto das outras commodities e debate suas características específicas; apresenta o Setor Elétrico Brasileiro (SEB) e o Mercado Brasileiro de Energia Elétrica e discute a Formação dos Preços no Mercado de Curto Prazo Brasileiro. Foram usados dados históricos para a estimação dos parâmetros de um modelo que capta as principais características dos preços spot de energia elétrica e, lançando mão do Método de Monte Carlo (MMC) para a simulação desses preços, foi analisada a flexibilidade de compra e venda parcial de um contrato de energia elétrica, usando a Teoria de Opções Reais (TOR). Concluiu-se que essa flexibilidade agrega valor aos contratos de energia.

\section{Palavras-chave}

Setor Elétrico Brasileiro; Comercialização de Energia Elétrica; Apreçamento de Contratos de Energia; Opções Reais; Método de Monte Carlo; Economia da Energia. 


\section{Abstract}

Abreu, Wagner Saboia de; Samanez, Carlos Patricio (Advisor); Baidya, Tara Keshar Nanda (Co-advisor). Modelling and Forecasting of Electricity Spot Prices and Applications within the Context of Investment under Uncertainty. Rio de Janeiro, 2012. 111p. MSc. Dissertation - Departamento de Engenharia Industrial, Pontifícia Universidade Católica do Rio de Janeiro.

The Brazilian Electric Power Industry (SEB) has undergone a major restructuring moving from a situation of state monopoly to a regulated privatization. In this process, interaction among agents took place in the industry, influencing electricity spot prices and consequently power derivative contracts. This work: places electric power in the context of other commodities and discusses its specific characteristics; presents the Brazilian Power Companies and the Brazilian Electricity Market and discusses the formation of short-term prices in Brazil. We used historical data to carry out the parameters' estimation of a model that captures the main characteristics of electricity spot prices and we analyzed a flexibility of partial buying or selling of one energy contract using the Real Options Approach (ROA), employing Monte Carlo Method (MCM) to simulate these prices. We concluded that this flexibility adds value to power contracts.

\section{Keywords}

Brazilian Electric Power Industry; Energy Marketing; Electricity Contracts Pricing; Real Options; Monte Carlo Method; Energy Economics. 


\section{Sumário}

$\begin{array}{ll}\text { 1. Introdução } & 15\end{array}$

1.1. Considerações Gerais $\quad 15$

$\begin{array}{ll}\text { 1.2. Motivação } & 18\end{array}$

$\begin{array}{lr}\text { 1.3. Objetivos } & 19\end{array}$

$\begin{array}{ll}\text { 1.4. Metodologia } & 19\end{array}$

1.5. Revisão Bibliográfica $\quad 24$

1.6. Organização do Trabalho 27

2. O Mercado de Energia Elétrica no Brasil 28

2.1. Considerações Iniciais 28

2.2. Alterações no Setor Elétrico Brasileiro 29

2.3. Instituições do Setor Elétrico Brasileiro 34

2.4. Comercialização de Energia no SEB 38

2.5. Formação dos Preços no Mercado de Curto

$\begin{array}{ll}\text { Prazo Brasileiro } & 42\end{array}$

3. Modelagem 53

3.1. Considerações Iniciais 53

3.2. Processos Estocásticos 55

3.3. Modelagem dos Preços da Eletricidade 68

3.4. Avaliação do Modelo 85

4. Opções Reais em Energia Elétrica 91

4.1. Considerações Iniciais 91 
4.3. Transação no Mercado Atacadista Brasileiro de Energia Elétrica

4.4. Opção de Escolha da Quantidade 95

4.4.1. Modelagem das Flexibilidades $\quad 95$

4.4.2. Penalidades 96

4.4.3. Opção de Escolha Parcial da Quantidade 97

4.4.4. Cálculo do Valor da Opção de Escolha Parcial da $\begin{array}{ll}\text { Quantidade } & 98\end{array}$

5. Conclusões e Oportunidades para Trabalhos Futuros 102

5.1. Conclusões 102

5.2. Oportunidades para Trabalhos Futuros 103

6. Referências Bibliográficas e Bibliografia 104

6.1. Referências 104

$\begin{array}{ll}\text { 6.2. Bibliografia } & 109\end{array}$ 


\section{Lista de Figuras}

Figura 1.1 - Preços de Curto Prazo de Algumas Commodities

Figura 1.2 - Quando as Opções Reais são Valiosas Incerteza x Flexibilidade

Figura 2.1 - Contabilização da Energia Disponível no Sistema

Figura 2.2 - Diagrama das Instituições do Sistema

Elétrico Brasileiro

Figura 2.3 - Processo de Decisão para Sistemas Hidrotérmicos

Figura 2.4 - Minimização do Custo de Operação de um

Sistema Hidrotérmico

Figura 2.5 - Custo Marginal de Operação x Volume Total de Produção 44

Figura 2.6 - Os Quatro Submercados do Sistema Elétrico Brasileiro $\quad 46$

Figura 2.7 - Armazenamento e Curva de Aversão ao Risco 51

Figura 3.1 - Preços Horários no Mercado de Curto Prazo (Nord Pool) 70

Figura 3.2 - Preço Mensal do Barril de Petróleo 1970-2000 71

Figura 3.3 - Comportamento Sazonal do Preço de Curto Prazo (Nord Pool)

Figura 3.4 - PLDs Semanais, Submercado: SE, Patamar de Carga

Médio

Figura 3.5 - Ln PLDs Semanais, Submercado: SE, Patamar de Carga Médio

Figura 3.6 - Coeficientes Dummy Mensais para Dessazonalização 82

Figura 3.7 - PLDs Semanais Dessazonalizados por Variáveis Dummy 83

Figura 3.8 - Regressão da eq. (45) 84

Figura 3.9 - Ajuste de Distribuição Exponencial de Dados 85

Figura 3.10 - Distribuições de ProbabilidadeSemanais do PLD 87

Figura 3.11 - PLD Semanal Realizado, Previsão e Erro de Previsão 88

Figura 4.1 - llustração da Modelagem da Opção de Escolha da Quantidade

Figura 4.2 - Valor da Opção de Escolha da Quantidade x

Flexibilidade(\%) 


\section{Lista de Tabelas}

Tabela 2.1 - Mudanças do Setor Elétrico Brasileiro 32

Tabela 2.2 - Patamares de Carga 45

Tabela 3.1 - Resumo das estatísticas descritivas do PLD e do In(PLD) 77

Tabela 3.2 - Resultados da Regressão da eq. (47) 82

Tabela 3.3 - Resultados da Regressão da eq. (51) 84

Tabela 3.4 - Resultados da aplicação das Eqs. (53 e 54) 84

Tabela 3.5 - Medidas Agregadas de Erros 90

Tabela 4.1 - Características de um Contrato Hipotético de Venda de Energia Elétrica 


\section{Símbolos, Acrônimos e Abreviaturas}

ACL - Ambiente de Contratação Livre

ACR - Ambiente de Contratação Regulada

ANEEL - Agência Nacional de Energia Elétrica

BEN - Balanço Energético Nacional

CAR - Curvas Bianuais de Aversão ao Risco

CCVE - Contratos de Compra e Venda de Energia

CEF - Certificado de Energia Firme

CFaR - Cash Flow at Risk

CMO - Custo Marginal de Operação

CONAMA - Conselho Nacional do Meio Ambiente

DNAEE - Departamento Nacional de Águas e Energia Elétrica

EaR - Earning at Risk

Eletrobrás - Centrais Elétricas Brasileiras S.A.

EPE - Empresa de Pesquisas Energéticas

GW - Giga Watt

GWh - Giga Watt-hora

IEA - International Energy Agency

kW - Kilo Watt

kWh - Kilo Watt-hora

MAE - Mercado Atacadista de Energia Elétrica

MMC - Método (ou Simulação) de Monte Carlo

MME - Ministério de Minas e Energia 
Mtep - Milhões de Toneladas Equivalentes de Petróleo

MW - Mega Watt

MWh - Mega Watt-hora

OECD - Organisation for Economic Co-operation and Development

OPEP - Organização dos Países Exportadores de Petróleo

ONS - Operador Nacional do Sistema Elétrico

PCH - Pequenas Centrais Hidrelétricas

PLD - Preço de Liquidação das Diferenças

PPA - Power Purchase Agreement

Proinfa - Programa de Incentivo às Fontes Alternativas de Energia Elétrica

SEB - Sistema Elétrico Brasileiro

SIN - Sistema Interligado Nacional

TIR - Taxa Interna de Retorno

TOR - Teoria de Opções Reais

TWh - Tera Watt-hora

VaR - Value at Risk

VP - Valor Presente

VPL - Valor Presente Líquido

W - Watt

Wh - Watt-hora 
"... If I have seen a little further it is by standing on the shoulders of Giants." 